\title{
Search-Based Functional Test Data Generation Using Data Metamodel
}

\author{
János Oláh and István Majzik \\ Department of Measurement and Information Systems \\ Budapest University of Technology and Economics \\ Magyar Tudósok körútja 2., Budapest, Hungary \\ \{janos.olah,majzik\}@mit.bme.hu
}

Software testing is the process of evaluating the quality of the software under test (SUT) by controlled execution, with the primary aim to reveal inadequate behavior. Despite the automation offered by modern development environments, the process of test data generation remains a largely manual task. In this paper we present a model-based approach for the generation of test data, using searchbased software engineering technique.

Search-based software engineering (SBSE) is the use of search-based optimization algorithms to software engineering problems. Software testing is probably the most important domain of SBSE 1]. Most studies, however, have applied SBSE for structural testing, while the use of such techniques for functional testing is rarely addressed. The main reason is probably the implicit nature of the software specifications and the complexity of their mapping to fitness functions.

The key ingredients for the application of search-based optimization to test data generation is the choice of candidate representation and the definition of the fitness function. In our proposed approach we utilize the metamodel constructed to describe the context of the SUT. Parts of the program data, their relations and the constraints among their values are represented by the metamodel. Candidate test data are model instances conforming to this metamodel.

We focus on functional specifications that describe the expected behaviour of the SUT in case of a certain context. In order to test the accomplishment of a specification, we need test data that contain particular configuration of objects as input described by the specification. We refer to these as context patterns, and our goal is to generate test data that contain these patterns. The fitness function formulated to guide the test data generation rewards model instances that cover the model patterns.

The iterative manipulation of instances and pattern matching in order to calculate the fitness are executed with popular model transformation frameworks, which usually apply rule based manipulation of models [2].

\section{References}

1. McMinn, P.: Search-based software test data generation: A survey. Software Testing, Verification and Reliability 14, 105-156 (2004)

2. Rozenberg, G. (ed.): Handbook of Graph Grammars and Computing by Graph Transformation, vol. 1. World Scientific, Singapore (1997) 